This item was submitted to Loughborough's Research Repository by the author.

Items in Figshare are protected by copyright, with all rights reserved, unless otherwise indicated.

\title{
A curve fitting methodology to determine impact location, timing, and instantaneous post-impact ball velocity in cricket batting
}

\section{PLEASE CITE THE PUBLISHED VERSION}

https://doi.org/10.1177/1754337117723275

\section{PUBLISHER}

SAGE Journals (@ IMechE)

VERSION

AM (Accepted Manuscript)

\section{PUBLISHER STATEMENT}

This work is made available according to the conditions of the Creative Commons Attribution-NonCommercialNoDerivatives 4.0 International (CC BY-NC-ND 4.0) licence. Full details of this licence are available at: https://creativecommons.org/licenses/by-nc-nd/4.0/

\section{LICENCE}

CC BY-NC-ND 4.0

\section{REPOSITORY RECORD}

Peploe, Chris, Stuart A. McErlain-Naylor, Andy Harland, Fred Yeadon, and Mark King. 2019. "A Curve Fitting Methodology to Determine Impact Location, Timing, and Instantaneous Post-impact Ball Velocity in Cricket Batting". figshare. https://hdl.handle.net/2134/25940. 


\title{
A curve fitting methodology to determine impact location, timing, and instantaneous post-impact ball velocity in cricket batting
}

\author{
${ }^{1}$ C. Peploe, ${ }^{1}$ S.A. McErlain-Naylor, ${ }^{2}$ A.R. Harland, ${ }^{1}$ M.R. Yeadon, and ${ }^{1}$ M.A. King \\ ${ }^{1}$ School of Sport, Exercise and Health Sciences, Loughborough University, Leicestershire, LE11 3TU, UK \\ ${ }^{2}$ Sports Technology Institute, Loughborough University, Leicestershire, LE11 3QF, UK
}

\begin{abstract}
This study aimed to develop a methodology for accurate determination of the impact location of a cricket ball on the bat face, as well as the identification of bat-ball contact timing and post-impact instantaneous ball velocity in a whole body kinematic data collection environment. Three-dimensional kinematic data of bat and ball were recorded during fourteen batting strokes; eight hitting a static ball and six against a bowling machine. Curves were fitted separately to the pre- and post-impact phases of the ball position data against time in three axes according to logarithmic equations determined from mechanical principles. Separate Fourier series models were similarly fitted to the four corners of the bat face against time during the downswing prior to ball impact. Time of impact for the dynamic ball trials was determined based upon the intersection of preand post-impact curves, with impact location calculated from ball and bat face curves at this time. $R^{2}$ values for the goodness of fit of the ball and bat curves averaged $0.99 \pm$ 0.04 and $1.00 \pm 0.00$ with root mean square errors of $7.5 \pm 2.6$ and $0.8 \pm 0.2 \mathrm{~mm}$, respectively. Calculated impact locations were assessed against measured impact locations derived from the impression imparted to a fine powder coating on the bat face, finding absolute differences of $6.4 \pm 4.2$ and $7.1 \pm 4.4 \mathrm{~mm}$ in the transverse and longitudinal axes of the bat, respectively. Thus, an automated curve fitting methodology enables the accurate determination of cricket bat-ball impact characteristics for use in experimental investigations.
\end{abstract}

Keywords: cricket, impact location, curve fitting, timing, velocity, kinematic data

\section{INTRODUCTION}

In cricket, the impact location of the ball on the bat face has a substantial effect on the subsequent post-impact ball trajectory. Impacts further from the sweetspot or longitudinal axis of the bat result in lower ball speeds (Bower, 2012), often causing the bat to twist and the ball to depart on unintended trajectories (Symes, 2006). Evidently, the impact location has a considerable influence on the success of the batting stroke, and thus is an important measure of success in cricket batting. Despite this, minimal research has been conducted to investigate methods for determining an accurate measure of ball impact location during a dynamic hitting motion.

Attempting to obtain an accurate measure of ball impact location on the face of a cricket bat presents the researcher with a number of challenges. Ball release speeds between 32.0 and $40.0 \mathrm{~m} \cdot \mathrm{s}^{-1}$ are common for international fast bowlers (Worthington et al., 2013), and while the ball slows down during its flight and contact with the pitch, it can still be expected to reach the batsman with 82 to $86 \%$ of its initial speed (32.8 - $34.4 \mathrm{~m} \cdot \mathrm{s}^{-1}$ for a $40.0 \mathrm{~m} \cdot \mathrm{s}^{-1}$ delivery), (James et al., 2004). Due to this high ball speed, short impact durations of approximately $1.0-1.5 \mathrm{~ms}$ (Symes, 2006) and the relatively low sample rates used in typical human motion capture studies $(250 \mathrm{~Hz}-300 \mathrm{~Hz})$, (Worthington et al., 2013; Peploe et al., 2014) the precise instant of impact is often missed by the motion capture system. These difficulties in ascertaining impact timing, as well as rapid marker movement and tracking errors 
caused by spin on the ball, make determination of the impact location of the ball on the bat face problematic.

As a consequence of these difficulties, many existing batting biomechanical studies (Elliott et al., 1993, Stretch et al., 1998, Stuelcken et al., 2005, Taliep et al., 2007, Cork et al., 2010) disregard impact location as a measure of success, often choosing to focus solely on pre-impact bat and post-impact ball velocity, thus decreasing the ability to determine the causes of batting success. A simple categorical measure of the quality of bat-ball contact was implemented and validated for use by Müller and Abernethy, (Müller and Abernethy 2006, 2008) consisting of live categorisation of each impact by a trained observer into good, bad, and no contact groups based on the resulting ball trajectory. This method was then used by Muller and Abernethy (2006) and Weissensteiner et al. (2011) to examine differences in interceptive ability between highly skilled and lesser skilled groups in a number of batting scenarios. While this approach provides researchers with a method by which they can quantify impact quality, it still provides minimal insight into the precise impact location of the ball on the bat face, merely indicating whether a batsman hit the ball on the face of the bat, on the edge, or missed it altogether.

In the only cricket studies to physically measure impact location, McKellar et al. (1998) and Stretch et al. (2004) placed a grid of piezoelectric sensors on the bat face in a $7 \times 23$ square grid. Although this allowed them to quantify impact location with high accuracy and resolution, the fitting process was complex and time consuming, and therefore cannot be applied to each participant's bat in turn, instead relying on a standard instrumented bat for testing. This is problematic, particularly in elite cricket, as players become heavily accustomed to a bat with specific mass and inertial properties. Thus, a standard instrumented bat may affect their swing mechanics. Impact location has been measured using pressure sensitive impact stickers (Hocknell, 2002) and high-speed video analysis (Knudson, 1991, 1993) in golf and tennis, respectively, however, these methods are time consuming in their application and processing stages.

In a study investigating the effects of off-centre impacts on racket and wrist kinematics in tennis, King et al. (2016) calculated the frame at which the ball was closest to the racket from motion capture data, and subsequently used positional data from this frame to transform the ball location into the local coordinate system of the racket, and hence estimated the impact location. Due to the longer impact durations found in tennis (4.4-6.2 ms), (Haake et al., 2003) as opposed to cricket $(1.0-1.5 \mathrm{~ms})$, (Symes 2006) impact is considerably less likely to occur between frames, thus reducing the possibility of errors in estimating impact timing and location. Indeed, when capturing at $\leq 250 \mathrm{~Hz}$, as in existing batting studies (Peploe et al., 2014; Stulcken et al., 2005; Taliep et al., 2007; Dias and Ferdinands 2010) or at $480 \mathrm{~Hz}$, as in the tennis study by King et al.,19 the interval between capture frames of $\geq 4 \mathrm{~ms}$ or $2.1 \mathrm{~ms}$, respectively, presents a high possibility of missing impact.

In the most relevant study published to date, Betzler et al. (2012) developed a method for determining the impact location of a stationary golf ball on the clubface of a driver. Five Qualysis motion capture cameras $(1000 \mathrm{~Hz})$ captured the position of one marker on the ball and three spherical reflective markers placed on top of the clubhead, which were in turn used to calculate the position of four virtual markers around the perimeter of the clubface. The clubface was then treated as part of a sphere with radius $254 \mathrm{~mm}$ to account for its curvature, and the exact timing of impact determined as the time at which the distance between the centre of the 
clubhead sphere and the ball centre dropped below $254 \mathrm{~mm}$ plus ball radius, using an iterative cubic best fit extrapolation of the clubhead position to calculate between frames. A similar extrapolation tool was used to determine the position of the virtual clubface markers at the time of impact, allowing impact location to be assessed. Direct comparison of the calculated impact location with the actual impact location, established using impact spray on the clubface, revealed mean offsets of $2.1 \pm 1.5$ and $1.7 \pm 1.4 \mathrm{~mm}$ in the horizontal and vertical directions of the clubface, respectively. In cricket batting, the pre-impact movement of the ball and the nonspherical nature of the bat face are not suitable for application of the method used by Betzler et al. (2012) for calculating impact timing and subsequent impact location. Any proposed methodology for use in cricket batting should also be applicable in a whole body kinematic data collection environment. Despite this, the method of Betzler et al. (2012) demonstrates the potential power of curve fitting and data extrapolation in attempting to calculate impact location.

The purpose of this study was to develop and validate a methodology for accurate determination of bat-ball impact location, as well as the timing of impact and instantaneous post-impact ball velocity using motion capture data in a whole body kinematic data collection environment. This will allow researchers to more accurately determine individual shot success through impact location and post-impact ball velocity, as well as more precisely identify and calculate joint and bat kinematics at the time of impact, some of which change rapidly during the downswing.

\section{METHODS}

All testing was conducted at the England \& Wales Cricket Board National Cricket Performance Centre, Loughborough, UK on a standard sized artificial cricket pitch. Data were recorded using an 18 camera Vicon Motion Analysis System (OMG Plc, Oxford, UK) operating at $250 \mathrm{~Hz}$. Testing procedures were explained to the participant involved, and informed written consent was obtained in accordance with the guidelines of the Loughborough University Ethical Advisory Committee. The participant completed a thorough self-selected warm-up before data collection.

\section{Stage 1 - Spatial reconstruction accuracy}

After standard wand calibration, an array of markers with fixed separation was passed through the capture volume to quantify the 3D spatial reconstruction accuracy of the motion capture system. The root mean square error (RMSE) from the mean marker separation was calculated for each pair as a measure of system noise, and compared to errors found later in the methodology.

\section{Stage 2 - Pre-impact ball tracking}

Data collection. Five $15 \times 15 \mathrm{~mm}$ squares of Scotchlite 7610 reflective tape (3M, Bracknell, UK) were attached to a standard size adult male cricket ball (Dukes Special County; Figure 1). Six $25 \mathrm{~mm}$ spherical reflective markers were also positioned on the top and base of the stumps at the batsman's end of the pitch. Finally, a rigid foam mat with impact paper taped to the front surface was positioned upright directly adjacent to the stumps. Nine trials were then completed, consisting of the ball being projected from a bowling machine (BOLA Professional, BOLA, UK), bouncing on the pitch, and impacting the mat placed adjacent to the stumps with a velocity of $22.6 \pm 1.1 \mathrm{~m} \cdot \mathrm{s}^{-1}$ (calculated using differentiated ball position data over a $36 \mathrm{~ms}$ interval). 


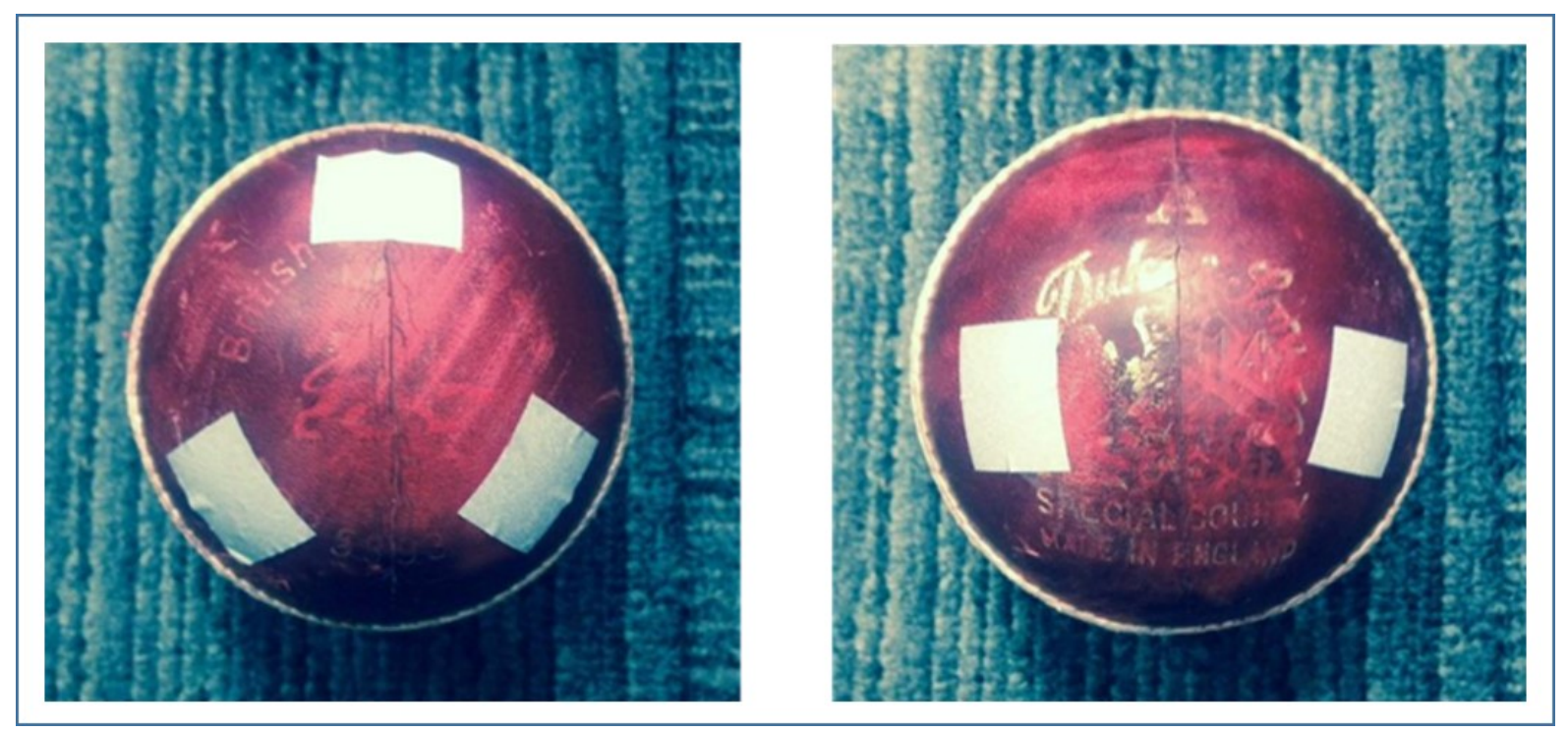

Figure 1. Reflective tape positioned on the cricket ball.

Data reduction. The ball and stumps marker position data were manually labelled and processed. Ball centre position was calculated on a frame-by-frame basis by taking the mean of ball marker positions in frames where three or more markers were reconstructed. Gaps in the ball centre position data at any given frame were then manually interpolated for the time point of that frame using a quintic spline tool in Vicon Nexus software. Curves were fitted to the unfiltered ball centre coordinate data for all points occurring after ball bounce (identified from a change in the vertical direction of the ball) and before impact with the mat (identified manually), separately against time in the anterior-posterior and medio-lateral axes according to Eq. (1), and in the vertical axis according to Eq. (2) (Figure 2; see Rationale for Treating Planar Accelerations Independently in Stage 4). Equations based on fundamental mechanical principles provide a more valid estimation of ball trajectories than straight lines or standard polynomials, and thus were chosen for this application. Ground contact was not included in the time period for which curves were fit to the ball coordinate data, so any deceleration in the horizontal directions (medio-lateral and anterior-posterior) was due solely to air resistance acting on the ball, with the addition of gravity in the vertical direction. Equation (1) was therefore derived for the anterior-posterior and medio-lateral directions from knowledge that the drag force acting on a body is proportional to the squared velocity of that body, and additionally that the deceleration of a body is proportional to the force acting on it (Appendix 1). Vertically, the addition of acceleration due to gravity led to the derivation of Eq. (2) (Appendix 2). Further assessment of the inherent assumptions in the application of these planar equations can be seen in Stage 4 of the present study. 


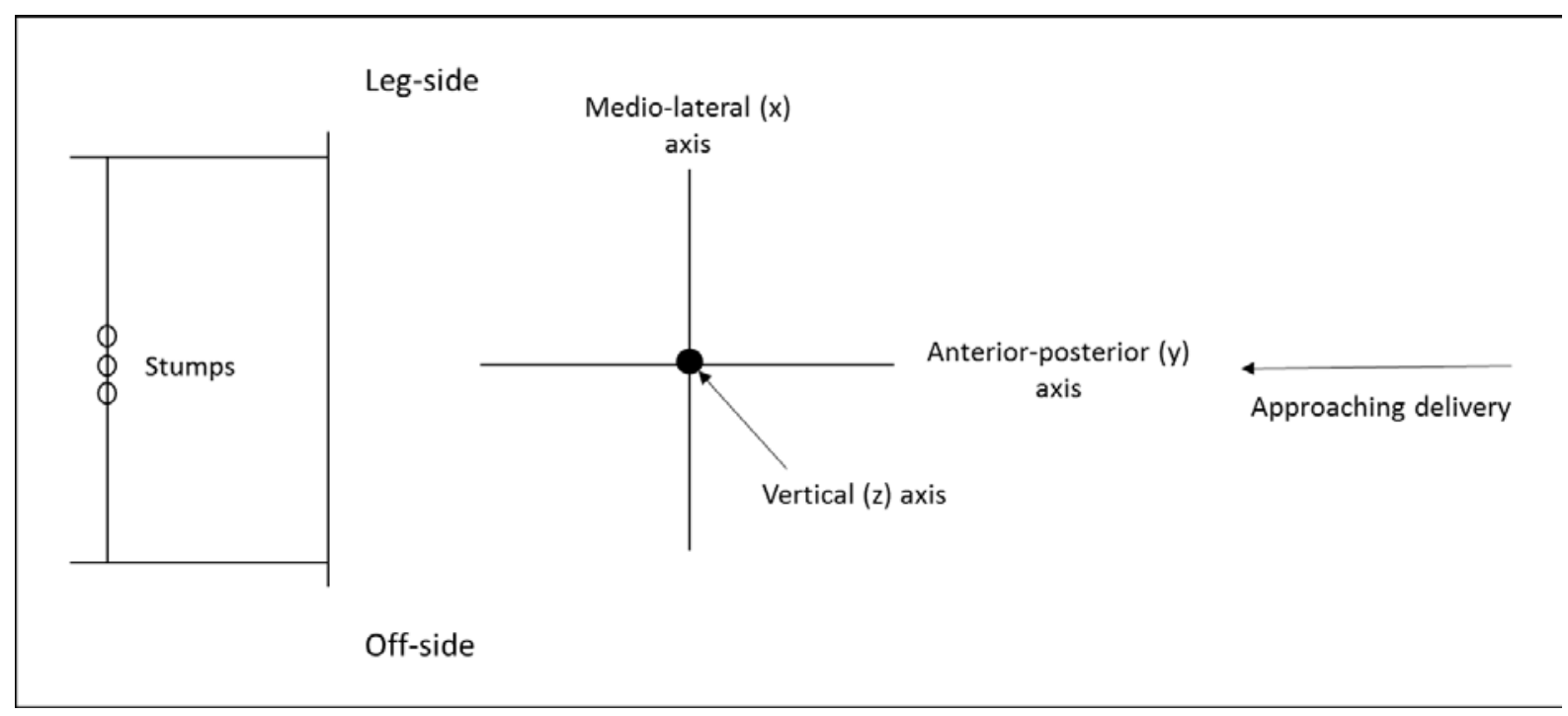

Figure 2. Global coordinate system.

$x=\frac{1}{k} \cdot \ln \left(1+k \cdot v_{0} \cdot t\right)$

$$
x=\frac{1}{2 k} \cdot \ln \cdot \frac{\left(g-k \cdot v_{0}^{2}\right)}{\left[g-k a^{2} \cdot \frac{\left(1-\frac{a+v_{0}}{a-v_{0}} \cdot e^{2 \cdot a \cdot k \cdot t}\right)^{2}}{\left(1-\frac{a+v_{0}}{a-v_{0}} \cdot e^{2 \cdot a \cdot k \cdot t}\right)^{2}}\right]}
$$

where $x=$ displacement; $t=$ time; $k$ and $v_{0}$ are constants; and $a=\sqrt{\frac{g}{k}}$

Curves were fitted in MATLAB (Version 8.0, The MathWorks Inc., Natick, MA, 2012) utilising a Trust-Region-Reflective Least Squares algorithm (More and Sorensen, 1983) to determine separate values for $k$ and $v 0$ for individual trials in each of the three axes, both pre- and post-impact. $\mathrm{R}^{2}$ and RMSE values, calculated from the difference at each time point, assessed the goodness of fit of the curves to the ball centre coordinate data in each axis. Time of impact was defined as the time at which the preimpact anterior-posterior curve, plotted against time, intersected with the plane of the mat (taken as the mean anterior-posterior position of the six stump markers). Finally, the calculated ball position at this time in the vertical and medio-lateral axes (from the curve equations) was compared to the digitised centre of the circular impact mark imparted to the impact paper (Image-Pro Analyser software; Media Cybernetics Inc., MD, USA), and the absolute discrepancy between the two measures was evaluated.

\section{Stage 3 - Impact location calculation: static ball}

Data collection. Five squares of reflective tape were attached to the cricket ball as in Stage 2 (Figure 1). Four $14 \mathrm{~mm}$ spherical reflective markers were positioned on the back corners of the blade of a short-handle adult cricket bat (Kookaburra Kahuna 1000; Figure 3). A participant (age 26 years, height $1.88 \mathrm{~m}$, mass $93.2 \mathrm{~kg}$ ) of premier league club standard performed eight batting shots (four forward drives and four pull shots) hitting a stationary ball suitably positioned on a batting tee. Impact location in 
each case was derived from the digitised impression imparted to a fine powder coating on the bat face (Figure 4).

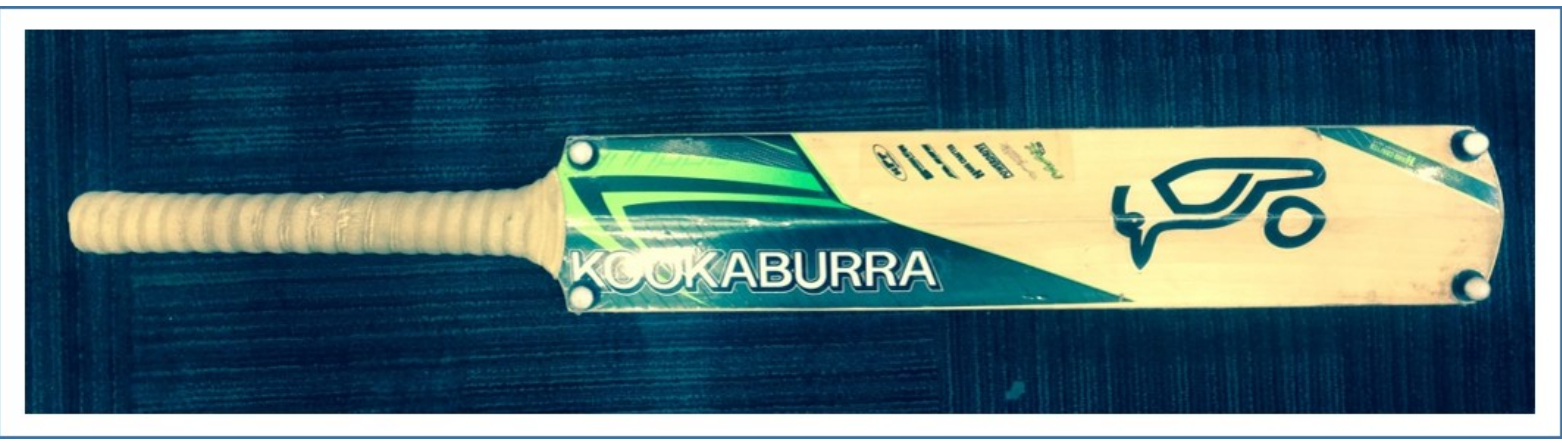

Figure 3. Reflective markers positioned on the blade of the cricket bat.

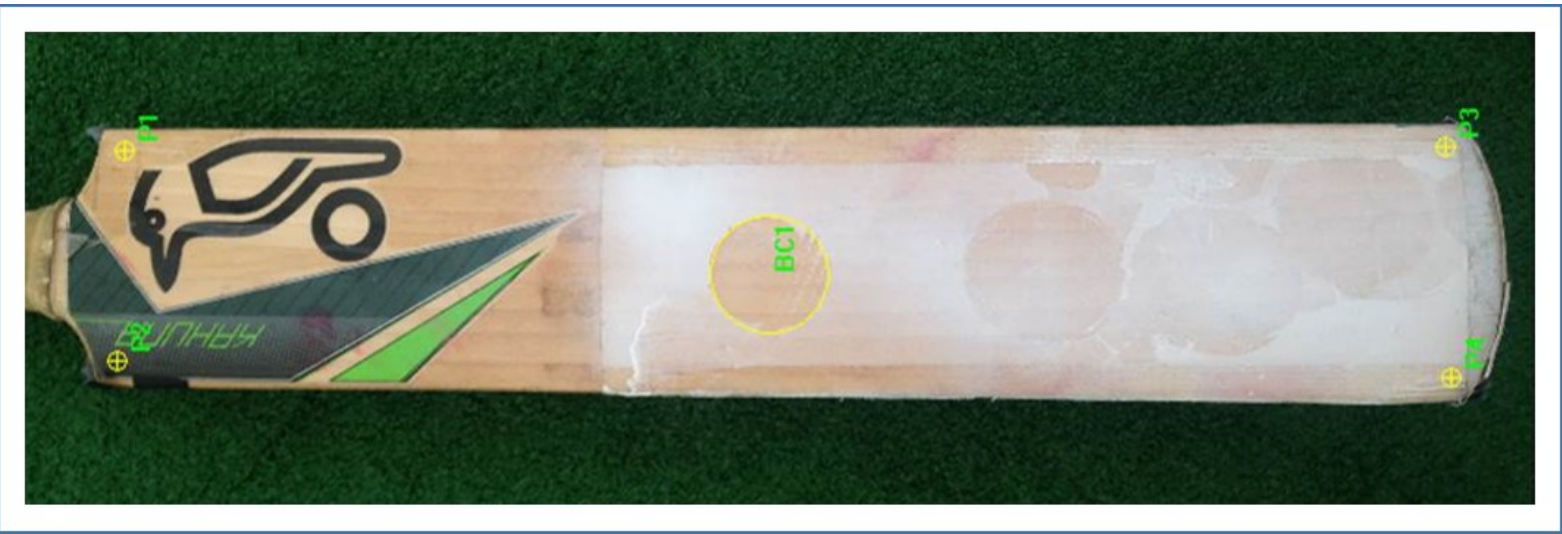

Figure 4. Impact location measurements derived from the impression imparted to a fine powder coating on the bat face.

Data reduction. The ball and bat marker position data were manually labelled and processed. The ball centre position was calculated and interpolated as described in Stage 2. Four virtual markers were created to define the bat face, based on the existing bat markers plus the measured depth of the bat plus marker radius in the normal axis of the bat's local coordinate system (longitudinal axis: toe to handle; normal axis: perpendicular to the bat face; transverse axis: parallel to the bat face running edge to edge; origin: bottom left corner of the bat face with the handle upwards). Any curvature or 'bowing' of the bat face was neglected due to their minimal magnitude and the bat face was represented by a flat rectangular plane.

Curves were fitted to the post-impact phase of the unfiltered ball centre coordinate data separately against time in the anterior-posterior and medio-lateral axes according to Eq. (1), and in the vertical axis according to Eq. (2). The postimpact phase was defined as data between impact (identified from a change in the anterior-posterior and medio-lateral position of the ball for the forward drive and pull shots, respectively) and either post-impact ball bounce (identified from a change in vertical direction of the ball) or the final frame before the ball exited the capture volume. Separate one-term Fourier models of displacement in the time domain were fitted to the four corners of the bat face (in the medio-lateral, anterior-posterior, and vertical axes) for $36 \mathrm{~ms}$ prior to the frame before impact. Four Fourier coefficients were determined for the model and subsequently used to obtain bat displacement at the specified impact time. Time of impact was defined as the time at which the postimpact ball curves, plotted against time, passed through the mean position of the ball centre during the static pre-impact phase (a $1 \mathrm{~s}$ period where the ball is stationary 
before impact) in its primary axis of motion (anterior-posterior for the forward drive, medio-lateral for the pull shot). Impact location was then calculated from the ball centre and bat face marker positions at the estimated impact time (from the curve equations) using global to local coordinate system rotation matrices, allowing the determination of the location of the ball centre relative to the local coordinate system of the bat.

The goodness of fit ( $R^{2}$ and RMSE) of each curve to the bat and ball centre coordinate data, and the difference between the measured and calculated impact locations in each axis were assessed as measures of accuracy within the methodology. Finally, knowledge of the post-impact curve equations enabled the calculation of resultant instantaneous post-impact ball velocity (Appendix 1 and 2). This was compared to post-impact ball velocity calculated via differentiation of ball position over a $36 \mathrm{~ms}$ time interval, in order to assess similarity between the two velocities, and thus validate the velocity derived from the curve fitting methodology.

\section{Stage 4 - Impact location calculation: dynamic ball}

Data collection. Reflective tape and markers were applied to the ball and bat respectively as described in Stage 3. The same participant performed six batting strokes (three forward drives and three pull shots) against the bowling machine with inbound velocity on the approach to impact of $24.1 \pm 0.7 \mathrm{~m} \cdot \mathrm{s}^{-1}$. Impact location in each case was again derived from the digitised impression imparted to a fine powder coating on the bat face (Figure 4).

Data reduction. The ball and bat marker position data were manually labelled and processed. The ball centre position was calculated and interpolated as in Stage 3. Curves were fitted to the pre- and post-impact phases of the unfiltered ball centre coordinate data separately against time in the anterior-posterior and medio-lateral axes according to Eq. (1) and in the vertical axis according to Eq. (2). The pre-impact phase was defined as data between any ball bounce and impact (identified from a change in the vertical and anterior-posterior directions of the ball respectively), while the post-impact phase was defined as in Stage 3. Separate Fourier series models were fitted to the four corners of the bat face as in Stage 3. Time of impact was defined as the mean time at which the pre- and post-impact ball curves, plotted against time, intersected in the vertical and anterior-posterior axes. Data in the mediolateral axis was not included in this section of the analysis due to the poor pre-impact $\mathrm{R}^{2}$ fit values (mean $0.50 \pm 0.49$; see Stage 4 Results) found while curve fitting in this axis. Although fitting a curve to this data only produced a small RMSE (6.48 \pm 2.4 $\mathrm{mm}$ ), the poor fit values were attributed to the fact that the magnitude of the tracking errors was at times greater than actual ball displacement in this axis, as the ball was projected almost entirely anterio-posteriorly and vertically with minimal displacement in the medio-lateral axis. Impact location was then calculated from the ball centre and bat face marker positions at the estimated impact time as in Stage 3.

The goodness of fit $\left(R^{2}\right.$ and RMSE) of each curve to the bat and ball centre coordinate data, the difference in estimated impact time between the vertical and anterior-posterior axes, and the difference between the measured and calculated impact locations in each axis were assessed. As in Stage 3, a comparison between post-impact ball velocity calculated via the curve fitting methodology and differentiation was conducted.

Rationale for treating planar accelerations independently. The retarding acceleration arising from the drag force acting on the ball will be proportional to the square of the velocity in the direction opposing that resultant velocity. Assuming that 
the cosine of the angle $\alpha$ between the velocity $v$ and the coordinate axis $x$ varies little over the period of flight considered, $\ddot{x}=-k_{x} v^{2}$ medio-laterally where $k_{x}=k \cos \alpha$ is assumed to be constant. Similarly, in the anterior-posterior (y) and vertical (z) axes, $\ddot{y}$ $=-\mathrm{k}_{\mathrm{y}} \mathrm{v}^{2}$ and $\ddot{z}=\mathrm{g}-\mathrm{k}_{\mathrm{z}} \mathrm{v}^{2}$ where $\mathrm{k}_{\mathrm{z}}=\mathrm{k} \cos \gamma$ and $\mathrm{k}_{\mathrm{y}}=\mathrm{k} \cos \beta$ are assumed to be constant.

In support of the assumption of constant $k_{x}, k_{y}$, and $k_{z}$, the ranges of cosa, $\cos \beta$, and cosy for the individual post-impact ball displacement curves were found to be $0.005 \pm 0.008,0.003 \pm 0.002$, and $0.035 \pm 0.018$, respectively. The minimum and maximum values during each trial are presented in Table 1 . Since the lift force is also proportional to $\mathrm{v}^{2}$, the same argument may be made for including the lift acceleration in the directions of the coordinate axes. As a consequence, the best fit constants $k_{x}$, $\mathrm{k}_{\mathrm{y}}$, and $\mathrm{k}_{\mathrm{z}}$ will include the effects of both lift and drag.

Table 1. Minimum and maximum values of $\cos \alpha, \cos \beta$, and $\cos \gamma$ for the individual post-impact ball displacement curves in Stage 4.

\begin{tabular}{|c|c|c|c|c|c|c|c|c|c|}
\hline $\begin{array}{l}\text { Trial } \\
\text { No. }\end{array}$ & $\begin{array}{c}\operatorname{Min} \\
\cos \alpha\end{array}$ & $\begin{array}{c}\operatorname{Max} \\
\cos \alpha\end{array}$ & Difference & $\begin{array}{c}\operatorname{Min} \\
\cos \beta\end{array}$ & $\begin{array}{c}\operatorname{Max} \\
\cos \beta\end{array}$ & Difference & $\begin{array}{c}\text { Min } \\
\cos Y\end{array}$ & $\begin{array}{l}\operatorname{Max} \\
\cos Y\end{array}$ & Difference \\
\hline 1 & 0.2287 & 0.2310 & 0.0023 & 0.9712 & 0.9733 & 0.0021 & 0.0189 & 0.0577 & 0.0388 \\
\hline 2 & 0.0247 & 0.0248 & 0.0000 & 0.9980 & 0.9997 & 0.0016 & 0.0044 & 0.0576 & 0.0531 \\
\hline 3 & 0.8483 & 0.8485 & 0.0002 & 0.5289 & 0.5291 & 0.0002 & 0.0128 & 0.0250 & 0.0121 \\
\hline 4 & 0.5301 & 0.5499 & 0.0198 & 0.3124 & 0.3150 & 0.0026 & 0.7736 & 0.7883 & 0.0147 \\
\hline 5 & 0.7464 & 0.7548 & 0.0084 & 0.6068 & 0.6136 & 0.0068 & 0.2320 & 0.2734 & 0.0415 \\
\hline 6 & 0.7087 & 0.7096 & 0.0009 & 0.7012 & 0.7053 & 0.0041 & 0.0170 & 0.0697 & 0.0527 \\
\hline Mean & & & 0.0053 & & & 0.0029 & & & 0.0355 \\
\hline SD & & & 0.0078 & & & 0.0023 & & & 0.0181 \\
\hline
\end{tabular}

\section{RESULTS}

\section{Stages 1 and 2 - Spatial reconstruction accuracy and pre-impact ball tracking}

RMSE values from the mean separation between the three marker pairs averaged $0.3 \pm 0.0 \mathrm{~mm}$. $\mathrm{R}^{2}$ and RMSE values for the goodness of fit of the preimpact ball curves averaged $0.97 \pm 0.05$ and $8.3 \pm 1.2 \mathrm{~mm}$, respectively (Table 2). Comparison of the calculated and measured ball impact locations on the mat resulted in mean absolute differences of $8.2 \pm 3.3$ and $3.6 \pm 2.1 \mathrm{~mm}$ in the medio-lateral and vertical axes, respectively. 
Table 2. Goodness of fit statistics for pre-impact ball curves in ball tracking trials (mean \pm SD).

\begin{tabular}{ccc}
\hline Global Axis & $\mathbf{R}^{2}$ & RMSE $(\mathbf{m m})$ \\
\hline Medio-lateral & $0.91 \pm 0.06$ & $7.6 \pm 2.5$ \\
Anterior-posterior & $1.00 \pm 0.00$ & $9.6 \pm 3.4$ \\
Vertical & $1.00 \pm 0.00$ & $7.6 \pm 2.1$ \\
\hline
\end{tabular}

\section{Stage 3 - Impact location calculation: static ball}

$\mathrm{R}^{2}$ and RMSE values for the goodness of fit of the post-impact ball curves averaged $0.95 \pm 0.11$ and $4.7 \pm 2.0 \mathrm{~mm}$, while those for the bat curves averaged $0.99 \pm$ 0.04 and $0.6 \pm 0.3 \mathrm{~mm}$, respectively (Table 3 ).

Table 3. Goodness of fit statistics for ball and bat curves in static ball impact location trials (mean \pm SD).

\begin{tabular}{ccc}
\hline Global Axis & $\mathbf{R}^{2}$ & RMSE (mm) \\
\hline Ball medio-lateral post & $0.99 \pm 0.03$ & $5.3 \pm 2.4$ \\
Ball anterior-posterior post & $1.00 \pm 0.01$ & $4.4 \pm 1.7$ \\
Ball vertical post & $0.87 \pm 0.18$ & $4.3 \pm 1.9$ \\
Bat medio-lateral pre & $0.98 \pm 0.08$ & $0.5 \pm 0.2$ \\
Bat anterior-posterior pre & $1.00 \pm 0.00$ & $0.7 \pm 0.4$ \\
Bat vertical pre & $1.00 \pm 0.00$ & $0.4 \pm 0.2$ \\
\hline
\end{tabular}

Note: pre/post relates to pre-/post-impact phase curves.

Comparison of the calculated and measured ball impact locations on the bat face resulted in mean absolute differences of $4.9 \pm 2.6$ and $4.6 \pm 2.7 \mathrm{~mm}$ in the transverse and longitudinal axes of the bat, respectively (Figure 5). Instantaneous post-impact ball velocity derived from the curve equations for the six impact location trials was $27.6 \pm 2.2 \mathrm{~m} \cdot \mathrm{s}^{-1}$. Comparison with ball velocity calculated via differentiation of post-impact ball position data over a $36 \mathrm{~ms}$ interval revealed an absolute difference of $0.3 \pm 0.2 \mathrm{~m} \cdot \mathrm{s}^{-1}$. 


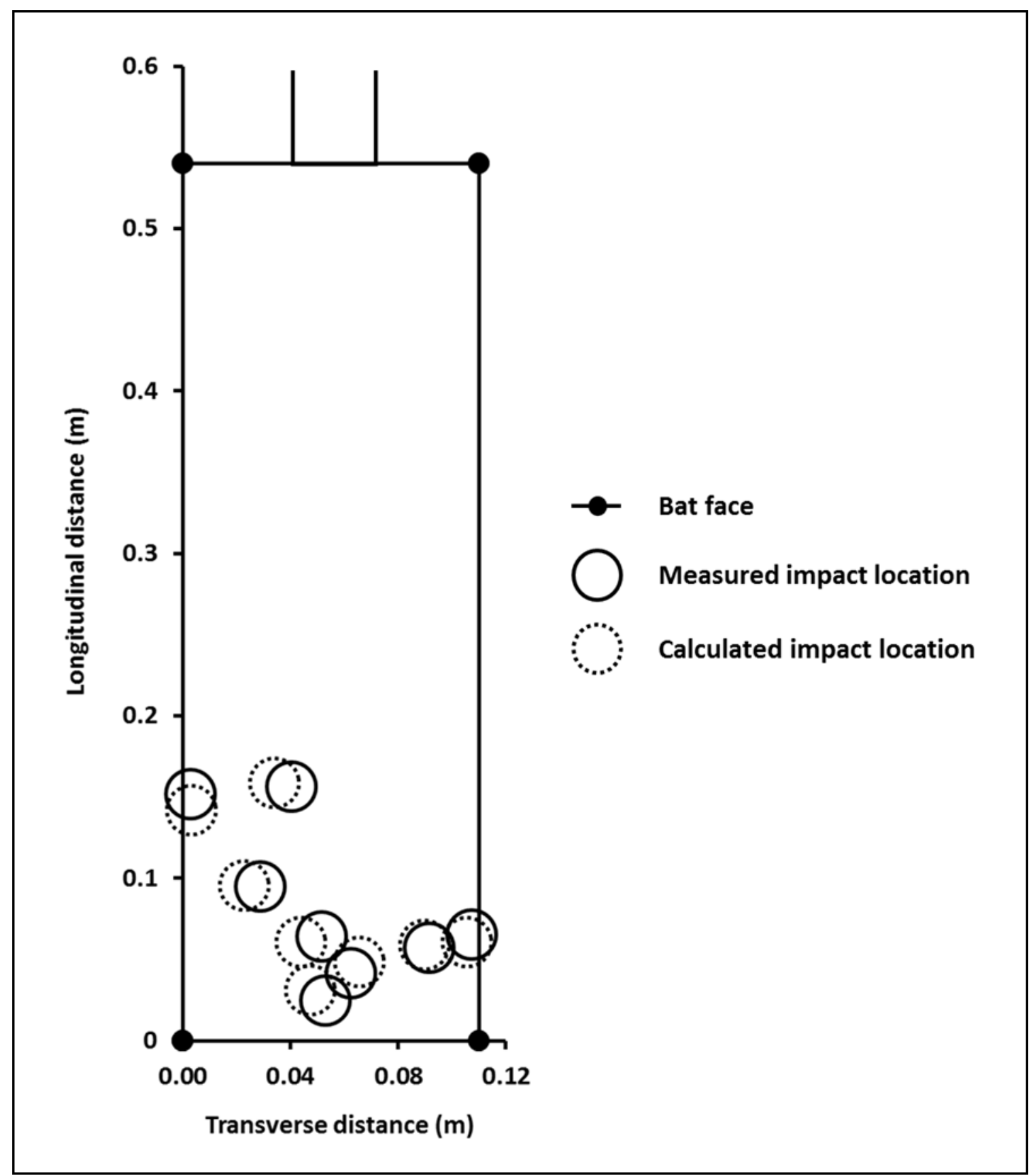

Figure 5. Measured and calculated impact locations in the static ball trials.

\section{Stage 4 - Impact location calculation: dynamic ball}

$\mathrm{R}^{2}$ and RMSE values for the goodness of fit of the pre- and post-impact ball curves (Fig. 6) averaged $0.90 \pm 0.26$ and $7.3 \pm 2.5 \mathrm{~mm}$, while those for the bat curves averaged $1.00 \pm 0.00$ and $0.8 \pm 0.2 \mathrm{~mm}$, respectively (Table 4). Removal of the preimpact medio-lateral ball curve fitting data, which was not used throughout the analysis, alters the mean $\mathrm{R}^{2}$ and RMSE values for those curves used in the methodology to 0.99 \pm 0.04 and $7.5 \pm 2.6 \mathrm{~mm}$, respectively. 


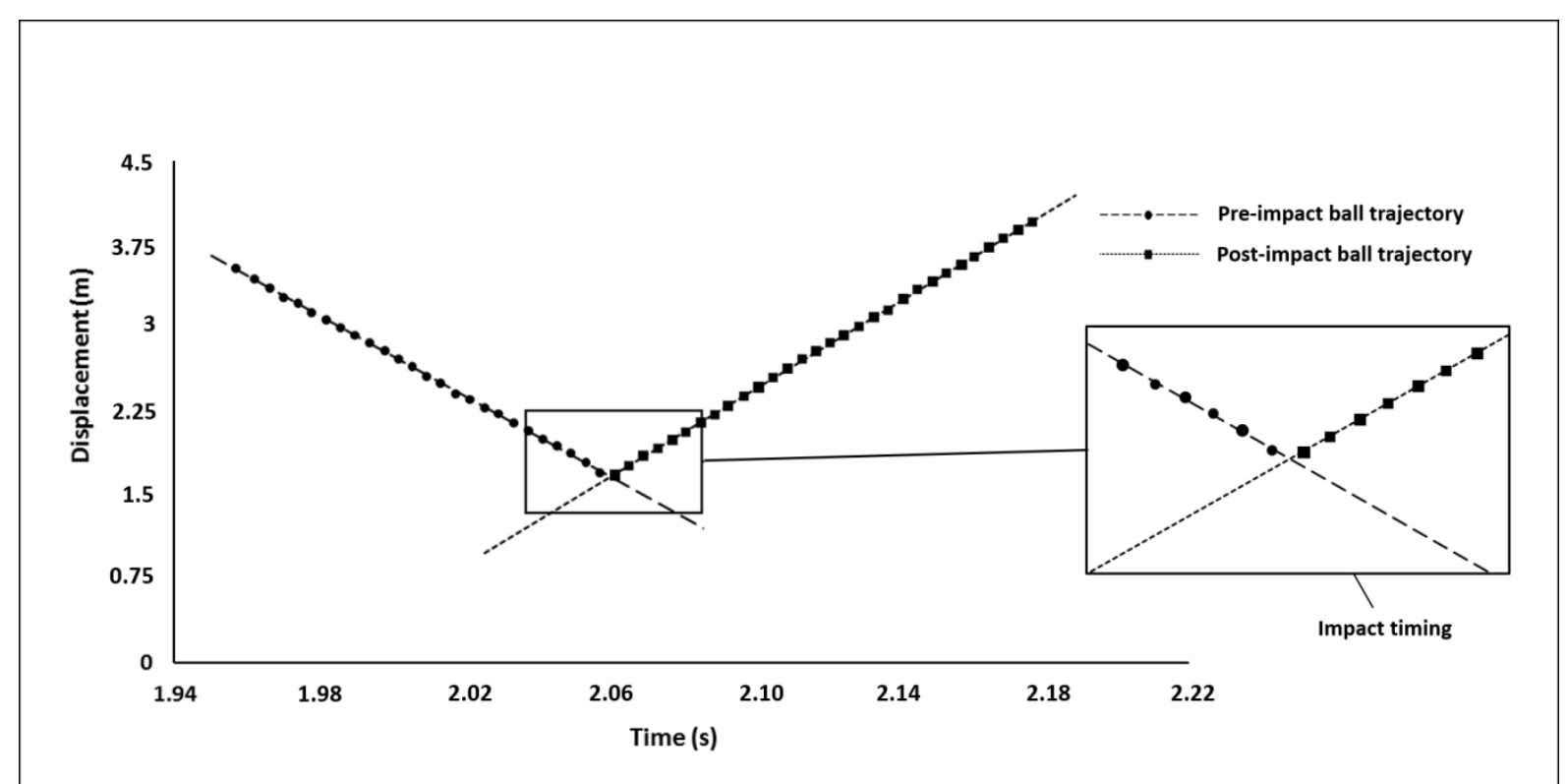

Figure 6. Pre- and post-impact ball curve fitting, and impact timing calculation in the anteriorposterior axis.

Table 4. Goodness of fit statistics for ball and bat curves in dynamic ball impact location trials (mean $\pm \mathrm{SD}$ ).

\begin{tabular}{ccc}
\hline Global Axis & $\mathbf{R}^{2}$ & RMSE $(\mathbf{m m})$ \\
\hline Ball medio-lateral pre & $0.50 \pm 0.49$ & $6.5 \pm 2.4$ \\
Ball medio-lateral post & $0.97 \pm 0.08$ & $8.1 \pm 2.9$ \\
Ball anterior-posterior pre & $1.00 \pm 0.00$ & $8.7 \pm 1.8$ \\
Ball anterior-posterior post & $1.00 \pm 0.00$ & $9.6 \pm 1.6$ \\
Ball vertical pre & $0.99 \pm 0.01$ & $6.2 \pm 1.7$ \\
Ball vertical post & $0.98 \pm 0.05$ & $4.8 \pm 1.7$ \\
Bat medio-lateral pre & $1.00 \pm 0.01$ & $1.0 \pm 0.5$ \\
Bat anterior-posterior pre & $1.00 \pm 0.00$ & $0.8 \pm 0.5$ \\
Bat vertical pre & $1.00 \pm 0.00$ & $0.5 \pm 0.3$ \\
\hline
\end{tabular}

Note: pre/post relates to pre-/post-impact phase curves.

Assessment of the estimated impact time (Figure 6) between curves in the vertical and anterior-posterior axes showed a mean difference of $1.7 \pm 1.4 \mathrm{~ms}$. Comparison of the calculated and measured ball impact locations on the bat face resulted in mean absolute differences of $6.4 \pm 4.2$ and $7.1 \pm 4.4 \mathrm{~mm}$ in the transverse and longitudinal axes of the bat, respectively (Figure 7). The calculated perpendicular distance from the bat face to the ball centre at the moment of impact was also evaluated and compared with the ball radius (35 $\mathrm{mm}$ excluding the seam), finding a mean absolute difference of $9.8 \pm 12.8 \mathrm{~mm}$. More detailed examination of the individual trials showed that one trial had a calculated impact location $44 \mathrm{~mm}$ from the midline. This impact was on the edge of the bat with the ball centre close to the plane of the bat face at the time of impact, which substantially affected this 
measurement. Removal of the results from this trial from the analysis reduced the absolute difference between the actual ball radius and the calculated perpendicular distance from bat face to ball centre at the moment of impact $(4.7 \pm 3.1 \mathrm{~mm})$. Instantaneous post-impact ball velocity derived from the curve equations for the six impact location trials was $21.9 \pm 4.7 \mathrm{~m} \cdot \mathrm{s}^{-1}$. Comparison with ball velocity calculated via differentiation of post-impact ball position data over a $36 \mathrm{~ms}$ interval revealed an absolute difference of $0.4 \pm 0.6 \mathrm{~m} \cdot \mathrm{s}^{-1}$.

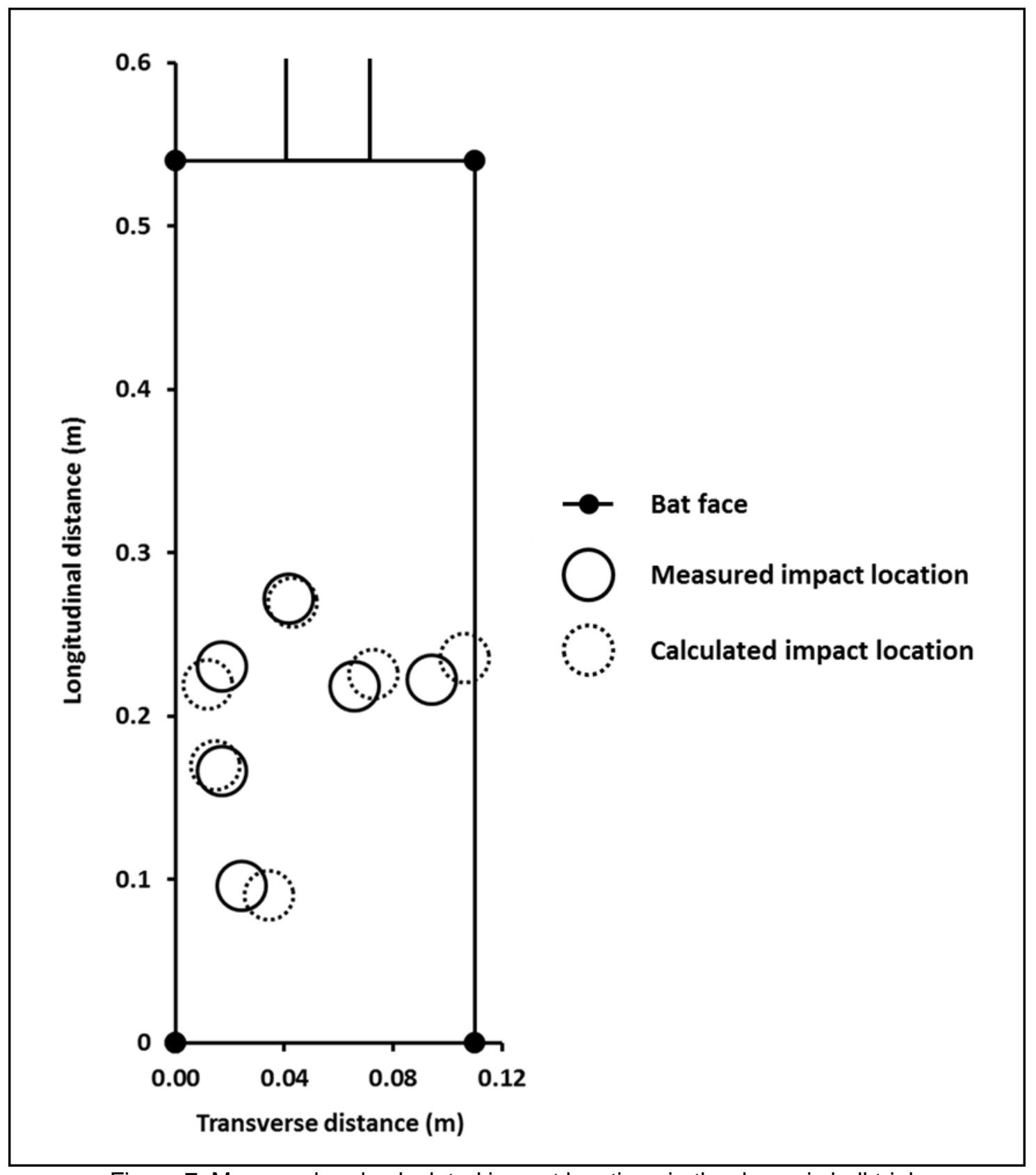

Figure 7. Measured and calculated impact locations in the dynamic ball trials

\section{DISCUSSION AND CONCLUSIONS}

A curve fitting methodology for the determination of the impact location of a cricket ball on a bat face, as well as the identification of bat-ball contact timing and post-impact instantaneous ball velocity, has been presented, and accuracy checks carried out for various steps of the process. Initial checks of the spatial reconstruction accuracy of the motion capture setup in Stage 1 revealed very small errors in marker 
separation with a mean RMSE of $0.3 \pm 0.0 \mathrm{~mm}$. This indicates that any larger errors found in later stages of the checking procedures are unlikely to be as a result of the reconstruction accuracy of the motion capture system, and are therefore likely to be a function of the methodology itself.

The high mean $\mathrm{R}^{2}$ and low mean RMSE values for all pre- and post-impact ball curves in Stages 2-4 justify the use of the logarithmic equations (Eqs. 1 and 2), and demonstrate that such equations are appropriate for modelling cricket ball trajectories shortly before and after impact. Any small errors in curve fitting, evidenced by nonperfect $\mathrm{R}^{2}$ and non-zero RMSE values, are most likely a result of errors in tracking the reflective tape positioned on the ball, or the ball centre calculation method when not all markers were visible. While the measured ball centre position is unlikely to correspond to the exact ball centre on a frame by frame basis, the fitting of logarithmic equations based on mechanical principles effectively reduces these errors, providing an accurate representation of ball trajectory during flight.

The lowest $\mathrm{R}^{2}$ values were consistently found in the medio-lateral axis of ball motion, with the mean pre-impact medio-lateral $R^{2}$ value for the six dynamic ball impact location trials in Stage 4 falling as low as 0.50 , thus justifying the removal of this data from the calculation of impact timing. This same curve, however, had a RMSE of $6.5 \mathrm{~mm}$, which was similar to the mean values across both the anteriorposterior and vertical axes. This highlights that the lower and more variable $\mathrm{R}^{2}$ values, found particularly in the pre-impact medio-lateral ball curves, were simply due to the small displacements in this axis. Ball displacements were much greater in the anterior-posterior and vertical axes for the majority of trials, and so the same absolute error (reflected by similar RMSE) resulted in lower $\mathrm{R}^{2}$ values for the medio-lateral curves. The same justification can be given to the slightly lower $\mathrm{R}^{2}$ values found in the post-impact vertical ball curves in Stage 3, caused by small displacement of the ball centre in this axis in certain trials.

All mean RMSE values for pre- and post-impact ball curves in Stages 2-4 were less than $10 \mathrm{~mm}$, indicating a high level of accuracy within the curve fitting procedure. The increased error magnitudes in comparison to measurements in Stage 1 are likely due to the added difficulty in accurately tracking the squares of tape positioned on a fast-moving spinning ball and errors created by the ball centre calculation methodology used. This is exemplified by the higher $\mathrm{R}^{2}$ and lower RMSE values found while fitting curves to the bat marker data, highlighting the relative simplicity in accurately tracking the position of markers travelling along a consistent trajectory in comparison to tape spinning around the circumference of a fast-moving ball.

Measured errors in calculated impact location on the mat in Stage 2 and on the bat in Stages 3 and 4 were found to be similar in magnitude to the RMSE values found in the pre- and post-impact ball curve fitting stages of the methodology. This indicates that no additional major errors were generated through the calculation of the timing of curve intersection with a plane or another curve, the inclusion of the bat face marker locations, or the transformation of position data from a global to local coordinate system, therefore suggesting that the largest absolute errors are found in ball tracking and ball centre calculation. Small errors in Stages 3 and 4 could also be attributed to the assumption that the bat represents a flat plane, when in reality the majority of bats exhibit a small amount of 'bowing' or curvature across their face which could slightly affect the accuracy of exact impact timing, and thus the impact location on the bat face. However, given the fact that the majority of the error in calculated impact location is accounted for by the errors in ball tracking (as highlighted by the similarity of the RMSE of the ball curves and the absolute measured error in impact location), 
strong evidence exists that the assumption of a flat bat face adds negligible additional error to the estimation of impact location, justifying it as a valid assumption.

The difference in estimated impact timing between the anterior-posterior and vertical axes in Stage 4 was $1.7 \pm 1.4 \mathrm{~ms}$. The magnitude of this difference can be partly explained by the presence of a single trial, in which the pre- and post-impact change in vertical ball displacement is markedly smaller than in the remainder of the trials. As a result of this small change in displacement, any tracking errors present in the ball trajectory have a relatively large effect on the quality of curve fitting, as shown by the lower $\mathrm{R}^{2}$ fit value in the vertical axis for this trial $(0.92 \pm 0.08)$ when compared to the remaining five trials $(1.00 \pm 0.00)$. When considering the time between frames in this study (4 ms) and the $1.0-1.5 \mathrm{~ms}$ impact duration between bat and ball in cricket (Symes, 2006) the magnitude of these differences in estimated impact timing indicate that the curve fitting methodology produces a more accurate estimation of the timing of impact and related impact location, rather than simply selecting the frame at which the ball centre is closest to the plane of the bat face (King et al., 2017). The proximity of the perpendicular distance from bat face to ball centre at the time of impact and the measured radius of a cricket ball in Stages 3 and 4 further enhance the credibility of the timing of impact calculations and calculated bat and ball position at this time.

When compared to differentiation over a 10-frame time interval (36 ms), the resultant velocities obtained via curve fitting in the Stage 3 and 4 impact location trials, revealed absolute differences of $0.3 \mathrm{~m} \cdot \mathrm{s}^{-1}$ and $0.4 \mathrm{~m} \cdot \mathrm{s}^{-1}$ respectively. While these differences do not indicate an error in either velocity measure due to inherent differences between the calculated average and instantaneous velocities, their similarity indicates a high level of accuracy in determining post-impact ball velocity from the curve fitting methodology.

It is important that future studies investigating the kinematics of batting in cricket obtain accurate data for impact timing, location, and post-impact ball velocity and trajectory, in order to more closely link kinematic parameters with the outcome success of the stroke. A curve fitting methodology enables this to be achieved to a greater degree of accuracy than the identification of the nearest frame to contact and differentiation methods used previously (King et al., 2017) and without the timeconsuming analysis required for quantification of impact location using high-speed video (Knudson, 1991, 1993) impact stickers (Hocknell, 2002) or piezoelectric sensors (Stretch et al., 2004). A more precise estimation of impact timing subsequently allows more accurate estimates of kinematics of important joints or segments at this time, particularly when considering fast moving joints, such as the elbow and wrist, or segments such as the bat, thus increasing the validity of any conclusions drawn from these measures.

The potential exists for future studies to select the complexity of the curve fitting methodology utilised to best fit their required accuracy. For example, a certain degree of mechanical accuracy can be sacrificed in favour of simplicity through the application of Eq. (1) to all three directions, including the vertical. The parameter $k$ is able to vary in the curve fitting process to account for the effects of gravity on the acceleration of the ball. Indeed, had Eq. (1) been applied in all directions in the present study, the $\mathrm{R}^{2}$ and RMSE values in the vertical axis would have been $0.98 \pm 0.04$ and $6.6 \pm 1.9 \mathrm{~mm}$, respectively, as opposed to $0.98 \pm 0.04$ and $5.5 \pm 1.8 \mathrm{~mm}$ in the full methodology. Similarly, the mean absolute differences between calculated and measured impact locations would have been $7.4 \pm 4.8 \mathrm{~mm}$ (medio-lateral) and $9.2 \pm 4.3 \mathrm{~mm}$ (longitudinal) as opposed to $6.4 \pm 4.2 \mathrm{~mm}$ (medio-lateral) and $7.1 \pm 4.4 \mathrm{~mm}$ (longitudinal) when the full methodology is applied. 
Although the curve fitting methodology has been applied here to cricket, this methodology may have applications across a range of hitting sports such as tennis, baseball, and badminton. The use of a curve equation based on fundamental mechanical principles of projectile flight allows more scope for future research to examine kinematics at exact points within ball flight, such as the time of ball bounce, where existing raw data may not be present. Researchers should, however, consider which axes are most appropriate for the calculation of impact timing in their given application. Trials with small displacements in a given axis (for example a pre-impact ball toss in a tennis serve may have little anterior-posterior or medio-lateral displacement) or no apparent change in direction (for example when missed or edged such that the post-impact trajectory is very similar to pre-impact) should be carefully assessed before interpreting further results. Likewise, researchers should consider the appropriateness of the stated assumptions when treating planar accelerations independently in alternative applications. As this study has identified the ball tracking and ball centre calculation stages of the methodology as the primary sources of error in determining impact location, future development should focus on more accurate methods of tracking and calculating the centre of a fast-moving spinning ball. This study has successfully developed and validated a methodology for the accurate determination of impact location of a cricket ball on the bat face, as well as the identification of bat-ball contact timing and post-impact instantaneous ball velocity, allowing researchers to more accurately determine shot success and estimate joint and bat kinematics at the point of impact.

\section{DECLARATION OF CONFLICTING INTERESTS}

The authors declare that there is no conflict of interest.

\section{FUNDING}

This project was part-funded by the England and Wales Cricket Board (ECB). 


\section{REFERENCES}

1. Bower R. The sweet spot of a cricket bat for low speed impacts. Sports Engineering 2012; 15 (2): 53-60.

2. Symes AW. The effect of mass distribution on cricket bat playing properties. PhD Thesis, Loughborough University, 2006.

3. Worthington PJ, King MA, and Ranson CA. Relationships between fast bowling technique and ball release speed in cricket. Journal of Applied Biomechanics 2013; 29 (1): 78-84.

4. James DM, Carré MJ, and Haake SJ. The playing performance of county cricket pitches. Sports Engineering 2004; 7 (1): 1-14.

5. Peploe C, King MA, and Harland AR. The effects of different delivery methods on the movement kinematics of elite cricket batsmen in repeated front foot drives. Procedia Engineering 2014; 72: 220-225.

6. Elliott B, Baker J, and Foster D. The kinematics and kinetics of the off-drive and on-drive in cricket. Australian Journal of Science and Medicine in Sport 1993; 25 (2): 48-54.

7. Stretch R, Buys FJ, Du Toit E, and Viljoen G. Kinematics and kinetics of the drive off the front foot in cricket batting. Journal of Sports Sciences 1998; 16 (8): 711-720.

8. Stuelcken MC, Portus MR, and Mason BR. Off-side front foot drives in men's high performance cricket. Sports Biomechanics 2005; 4 (1): 17-35.

9. Taliep MS, Galal U, and Vaughan CL. The position of the head and centre of mass during the front foot off-drive in skilled and less-skilled cricket batsmen. Sports Biomechanics 2007; 6 (3): 345-360.

10. Cork A, Justham $L$, and West AA. Batter's behaviour during training when facing a bowling machine and when facing a bowler. Proceedings of the Institution of Mechanical Engineers, Part P: Journal of Sports Engineering and Technology 2010; 224 (3): 201-208.

11. Müller $S$ and Abernethy B. Batting with occluded vision: An in-situ examination of the information pick-up and interceptive skills of high-and lowskilled cricket batsmen. Journal of Science and Medicine in Sport 2006; 9 (6): 446-458.

12. Müller $S$ and Abernethy B. Validity and reliability of a simple categorical tool for the assessment of interceptive skill. Journal of Science and Medicine in Sport 2008; 11 (6): 549-552.

13. Weissensteiner JR, Abernethy B, and Farrow D. Hitting a cricket ball: what components of the interceptive action are most linked to expertise? Sports Biomechanics 2011; 10 (4): 324-338.

14. McKellar DK, Nurick GN, and Stretch RA. The measurement of the position of a ball striking a cricket bat. The Engineering of Sport: Design and Development 1998; 1: 275-282.

15. Stretch RA, Nurick GN, Balden V, and McKellar DK. The position of impact of a ball striking a cricket bat: assisting coaches with performance analysis of cricket technique and skill levels. International Journal of Performance Analysis in Sport 2004; 4 (2): 74-81.

16. Hocknell A. High-performance driver design: benefits for all golfers. Journal of Sports Sciences 2002; 20 (8): 643-649.

17. Knudson D. Effect of string type and tension on ball vertical angle of rebound in static tennis impacts. Journal of Human Movement Studies 1991; 20: 3947. 
18. Knudson D. Effect of string tension and impact location on ball rebound accuracy in static tennis impacts. Journal of Applied Biomechanics 1993; 9 (2): 143-148.

19. King MA, Hau A and Blenkinsopp G. The effect of ball impact location on racket and forearm joint angle changes for one-handed tennis backhand groundstrokes. Journal of Sports Sciences 2016; Published online: July 27, 2016.

20. Haake SJ, Carré MJ, and Goodwill SR. The dynamic impact characteristics of tennis balls with tennis rackets. Journal of Sports Sciences 2003; 21 (10): 839-850.

21. Dias GR and Ferdinands R. The biomechanics of the initial movement in cricket batting. In: Portus M (ed) Cricket Australia Conference of Science and Medicine in Coaching, Conference Proceedings, 1-3 June 2010: 63-66.

22. Betzler NF, Monk SA, Wallace ES, and Otto SR. Variability in clubhead presentation characteristics and ball impact location for golfers' drives. Journal of Sports Sciences 2012; 30 (5): 439-448.

23. Moré JJ and Sorensen DC. Computing a trust region step. SIAM Journal on Scientific and Statistical Computing 1983; 4 (3) 553-572. 


\section{APPENDIX 1: CRICKET BALL DISPLACEMENT EQUATION}

The cricket ball decelerates during flight (both pre- and post-impact), and can be expected to reach the batsman with 82 to $86 \%$ of its initial speed (James et al., 2004). Ground contact was not included in the time period for which curves were fitted to the ball coordinate data in the present study and so any deceleration was due to air resistance acting on the ball. The drag force acting on a body is proportional to the squared velocity of that body and additionally, the deceleration of a body is proportional to the force acting on it ( $\mathrm{x}$ : displacement; $\mathrm{v}$ : velocity; t: time):

$$
\begin{gathered}
v \cdot \frac{d v}{d x}=-k \cdot v^{2} \\
-\frac{d v}{v}=k \cdot d x \\
-\int_{v_{0}}^{v} \frac{d v}{v}=\int_{0}^{x} k \cdot d x,
\end{gathered}
$$

Where $v_{0}$ is the initial velocity at $x=0$ (the beginning of the curve). Thus:

$$
\begin{aligned}
\ln \left(\frac{v_{0}}{v}\right) & =k \cdot x \\
\frac{v_{0}}{v} & =e^{k x} \\
v & =v_{0} \cdot e^{-k x} \\
\frac{d x}{d t} & =v_{0} \cdot e^{-k x} \\
\int_{0}^{x} e^{k x} \cdot d x & =\int_{0}^{t} v_{0} \cdot d t \\
\frac{1}{k}\left(e^{k x}-1\right) & =v_{0} \cdot t \\
e^{k x} & =1+k \cdot v_{0} \cdot t .
\end{aligned}
$$

Thus:

$$
x=\frac{1}{k} \cdot \ln \left(1+k \cdot v_{0} \cdot t\right)
$$

Differentiating this curve with respect to time gives the instantaneous velocity:

$$
v=\frac{v_{0}}{1+k \cdot v_{0} \cdot t}
$$




\section{APPENDIX 2: CRICKET BALL VERTICAL DISPLACEMENT EQUATION}

In addition to the effects of drag forces as detailed in Appendix 1, the vertical trajectory of an airborne object such as a cricket ball is also dependent upon the acceleration due to gravity. As such acceleration in this axis is proportional to both the gravitational force and the drag force:

$$
\begin{aligned}
\frac{d v}{d t} & =g-k \cdot v^{2} \\
\frac{d v}{g-k \cdot v^{2}} & =d t \\
\frac{d v}{\frac{g}{k}-v^{2}} & =k \cdot d t
\end{aligned}
$$

Let:

$$
\frac{g}{k}=a^{2}
$$

Thus:

$$
\int_{v_{0}}^{v} \frac{d v}{a^{2}-v^{2}}=\int_{0}^{t} k \cdot d x
$$

Where $v_{0}$ is the initial velocity at $x=0$ (the beginning of the curve). Thus:

$$
\begin{aligned}
\frac{1}{2 a} \int \frac{(a-v)+(a+v)}{(a-v) \cdot(a+v)} \cdot d v & =k \cdot t \\
\frac{1}{2 a}\left[\ln \cdot \frac{a+v}{a-v}\right]_{v_{0}}^{v} & =k \cdot t \\
\frac{1}{2 a}\left[\ln \cdot \frac{a+v}{a-v}-\ln \cdot \frac{a+v_{0}}{a-v_{0}}\right]_{v_{0}}^{v} & =k \cdot t \\
\frac{1}{2 a}\left[\ln \cdot \frac{a+v}{a-v}-\ln \cdot \frac{a+v_{0}}{a-v_{0}}\right]_{v_{0}}^{v} & =k \cdot t \\
\frac{1}{2 a}\left[\ln \cdot \frac{a+v}{a-v} \cdot \frac{a-v_{0}}{a+v_{0}}\right]_{v_{0}}^{v} & =k \cdot t \\
\frac{a+v}{a-v} & =\frac{a+v_{0}}{a-v_{0}} \cdot e^{2 \cdot a \cdot k \cdot t}
\end{aligned}
$$




$$
\begin{aligned}
(a-v) \cdot\left[\frac{a+v_{0}}{a-v_{0}} \cdot e^{2 \cdot a \cdot k \cdot t}\right] & =a+v \\
v\left[1+\frac{a+v_{0}}{a-v_{0}} \cdot e^{2 \cdot a \cdot k \cdot t}\right] & =-a\left[1-\frac{a+v_{0}}{a-v_{0}} \cdot e^{2 \cdot a \cdot k \cdot t}\right] \\
& =\frac{-a\left[1-\frac{a+v_{0}}{a-v_{0}} \cdot e^{2 \cdot a \cdot k \cdot t}\right]}{\left[1-\frac{a+v_{0}}{a-v_{0}} \cdot e^{2 \cdot a \cdot k \cdot t}\right]}
\end{aligned}
$$

This equation enables instantaneous vertical velocity to be calculated. Substituting this equation for velocity into an equation derived for displacement also gives the final equation for vertical ball displacement:

$$
\begin{aligned}
\frac{d v}{d t} & =g-k \cdot v^{2} \\
\int_{v_{0}}^{v} \frac{v \cdot d v}{g-k \cdot v^{2}} & =\int_{0}^{x} d x \\
x & =-\frac{1}{2 k}\left[\ln \left(g-k \cdot v^{2}\right)\right]_{v_{0}}^{v} \\
x & =\frac{1}{2 k} \cdot \ln \cdot \frac{g-k_{v 0}^{2}}{g-k v^{2}}
\end{aligned}
$$

Thus:

$$
x=\frac{1}{2 k} \cdot \ln \cdot \frac{\left(g-k \cdot v_{0}^{2}\right)}{\left[g-k a^{2} \cdot \frac{\left(1-\frac{a+v_{0}}{a-v_{0}} \cdot e^{2 \cdot a \cdot k \cdot t}\right)^{2}}{\left(1-\frac{a+v_{0}}{a-v_{0}} \cdot e^{2 \cdot a \cdot k \cdot t}\right)^{2}}\right]}
$$

Where:

$$
a=\sqrt{\frac{g}{k}}
$$

